\title{
Special Issue on Physical Literacy: Evidence and Intervention
}

Welcome to this special issue of the Journal of Teaching in Physical Education (JTPE) that we believe provide readers with new data driven and empirical insight into the concept of physical literacy. This issue will have meaningful implications for the planning, reporting, assessment, and teaching of quality physical education in schools around the world. Authors contributing to this issue have provided new and the first robust evidence into origins of physical literacy, its validity, and how it can be applied into evidence-based teaching practice for a range of school-aged populations.

Based on an adaptation of the United Nations Educational, Scientific and Cultural Organization's definition of "literacy," "physical literacy" can be defined as:

The ability to move with confidence and competence using all the physical assets one has at their disposal at any given point in time across varying contexts. It involves a continuum of learning by enabling individuals to achieve their goals; to develop their knowledge, movement, and potential; and to participate fully in their community and wider society (Dudley, Cairney, Wainwright, Kriellaars, \& Mitchell, 2017, p. 6)

As physical educators we know that students need to actively participate in their learning and make decisions in physical education that help determine the trajectory of lifelong healthy living, social participation, and active citizenship. For this reason, quality physical education experiences must be informed by the philosophy and evidence being generated from empirically driven physical literacy research. Of course, this statement itself reveals a specific epistemological stance, one rooted in a positivistic tradition of empirically based inquiry. This has not been the only tradition evident in the field of physical literacy. Edwards et al. (2018) identified two "solitudes"- the pragmatic approach and the idealist approach - which they argue define the two foundations of thought under which previous research and theoretical papers in the field can be classified. Although a previous special issue of this journal (JTPE, 2018, volume 37, issue 3) tended toward the idealist perspective, this is the first to give due consideration to the pragmatic approach. We thank the editors of the JTPE for the opportunity to highlight this perspective.

A total of 10 articles that reported data from physical literacy studies that impact on our work as physical educators have been published in this special issue of the JTPE. One article traces the evolution of the physical literacy construct back to inception (Cairney, Kiez, Roetert, \& Kriellaars, 2019); two articles report findings on their respective Delphi data collection and analyses in attempts to operationalize physical literacy in their respective educational and policy structures (Keegan et al., 2019; Shortt, Webster, Keegan, Egan, \& Brian, 2019); one article, for the first time in the history, provides an empirical validation of the physical literacy construct (Cairney, Clark, Dudley, \& Kriellaars, 2019); another article provides something of a "hitchhikers guide" to selecting appropriate measures of physical literacy (Barnett et al., 2019); another article reports on the outcomes of a professional development program for primary school teachers in their operationalization of physical literacy (Edwards et al., 2019); and the remaining four articles report findings from research methods using an assessment (Mandigo, Treadway, \& Lodewyk, 2019), visually impaired students (Brian et al., 2019), young children (Gu, Chen, \& Xianxia, 2019), and the circus arts as intervention medium (Kriellaars et al., 2019) to explore the development of physical literacy in schools.

Our first article by John Cairney and colleagues is a comprehensive review into the origins of the physical literacy construct. The authors find writings referencing the concept of physical literacy occurring as early as the late 1800 s and into the early 20 th century prior to the Whiteheadian definitions dominating the discourse. They conclude that by discussing the anxieties that have arisen with the construction of the term and the conflation of the term with fitness or physical education, greater multidisciplinary potential of this construct is enabled. The second article in this special issue is ground breaking. Also led by Cairney from the University of Toronto with Canadian and Australian researchers, they report on the very first known construct validation of physical literacy. Using three different models and two samples comprising of over 2,000 students, they tested whether physical literacy can capture concepts such as motor competence, perceived competence, motivation, and enjoyment in Grade 5 and Grade 7 student populations.

The next two articles, one from Australia and the other from the United States, report on the Delphi processes that these countries have undertaken to operationalize physical literacy for educative purposes. The U.S. paper by Chelsee Shortt and colleagues operationalized physical literacy as an autonomous application of movement, constructed by the individual's conception of movement and response to adversity. This has important implications for planning and programming considerations physical educators use in their practices. The Australian paper led by Richard Keegan reports on the development of a physical literacy definition and standards framework suitable for implementation in that country. Importantly for physical educators, the researchers were able to firmly place physical literacy in the sphere of a "learning construct" underpinned by learning theory and progression that again will have meaningful implication for our work as teachers.

Following on from Keegan and colleagues Delphi paper, is another study from the Australian group led this time by Lisa Barnett that provides further guidance for physical educators in their assessment of physical literacy. In a similar fashion to an earlier "hitchhikers guide" to assessing physical activity by Dollman et al. (2009), Barnett and colleagues offer us key considerations and attributes to physical literacy assessment. It includes a simple to use decision flowchart that has also been reported to assist teachers to select appropriate methods of assessing physical literacy. A particularly nice feature is the inclusion of school physical education and sport scenarios to illustrate this process. 
From Wales, we see the important and necessary issue of teaching teachers about physical literacy addressed head-on. Lowri Edwards leads her team on reporting what it takes to implement a professional development program with primary/elementary school teachers to operationalize physical literacy in their classes. Although necessary and successful, the authors report many of the obstacles and issues with this process.

We also take pleasure in reading about some of the early interventions taking place in physical literary research. Using the "Passport for Life" assessment tool, James Mandigo and colleagues provide some early evidence as to the efficacy of the Teaching Games for Understanding model (Bunker \& Thorpe, 1982) in developing certain components of a child's physical literacy. Xiangli Gu and colleagues report on cross-sectional data measures associated with early U.S. interpretations of physical literacy measurement to show that discrepancies arise on a number of individual and group variables such as gender, weight, and ethnicity. Another interesting article by Ali Brian and colleagues sought to tackle the difficult question of physical literacy evident in children and adolescents with visual impairment. In this sample of young people, the authors were able to clearly argue for physical educators to focus on fostering motivation if they wished to improve physical literacy in visually impaired students.

The final paper led by Dean Kriellaars at the University of Manitoba reports on the novel effect of teaching circus arts to children and the impact it has on their physical literacy. Using a quasi-experimental design, the researchers were able to comprehensively demonstrate significant improvements in motor competence, children's confidence and comprehension of movement terminology, and active participation. The gap in motor competence between girls and boys in the circus arts intervention was also smaller.

We would like to thank all of the authors and the reviewers for their important contributions to this special issue on data and evidence in physical literacy. In addition, we also would like to thank Dr. Weidong Li for his confidence in us as guest editors and Dr. Mark Byra for his support and assistance throughout the process. We believe that this issue will add to the research base on physical literacy in a significant and meaningful way.

Dean Dudley Macquarie University John Cairney University of Toronto Jackie Goodway The Ohio State University

\section{References}

Barnett, L.M., Dudley, D., Telford, R.D., Lubans, D.R., Bryant, A., Roberts, W., . . Keegan, R. (2019). Guidelines for the selection of physical literacy measures in physical education in Australia. Journal of Teaching in Physical Education, 38. doi:10.1123/jtpe.2018-0219
Brian, A., De Meester, A., Klavina, A., Irwin, J.M., Taunton, S., Pennell, A., \& Lieberman, L. (2019). Exploring children with visual impairments' physical literacy: A preliminary investigation of autonomous motivation. Journal of Teaching in Physical Education, 38. doi:10.1123/jtpe.2018-0194

Bunker, D., \& Thorpe, R. (1982). A model for the teaching of games in secondary schools. The Bulletin of Physical Education, 18(1), $5-8$.

Cairney, J., Clark, H., Dudley, D., \& Kriellaars, D. (2019). Physical literacy in children and youth: A construct validation study. Journal of Teaching in Physical Education, 38. doi:10.1123/jtpe.2018-0270

Cairney, J., Kiez, T., Roetert, E.P., \& Kriellaars, D. (2019). A 20th century narrative on the origins of the physical literacy construct. Journal of Teaching in Physical Education, 38. doi:10.1123/jtpe.2018-0072

Dollman, J., Okely, A.D., Hardy, L., Timperio, A., Salmon, J., \& Hills, A.P. (2009). A hitchhiker's guide to assessing young people's physical activity: Deciding what method to use. Journal of Science and Medicine in Sport, 12, 518-525. PubMed ID: 19038579 doi:10. 1016/j.jsams.2008.09.007

Dudley, D., Cairney, J., Wainwright, N., Kriellaars, D., \& Mitchell, D. (2017). Critical considerations for physical literacy policy in public health, recreation, sport, and education agencies. Quest, 69, 436-452. doi:10.1080/00336297.2016.1268967

Edwards, L., Bryant, A., Morgan, K., Cooper, S.-M., Jones, A., \& Keegan, R. (2019). A professional development program to enhance primary school teachers' knowledge and operationalization of physical literacy. Journal of Teaching in Physical Education, 38. doi:10.1123/jtpe.2018-0275

Edwards, L.C., Bryant, A.S., Keegan, R.J., Morgan, K., Cooper, S.M., \& Jones, A.M. (2018). Measuring physical literacy and related constructs: A systematic review of empirical findings. Sports Medicine, 48, 659-682. PubMed ID: 29143266 doi:10.1007/s40279-0170817-9

Gu, X., Chen, S., \& Xianxia, Z. (2019). Physical literacy at the start line: Young children's motor competence, fitness, physical activity, and fitness knowledge. Journal of Teaching in Physical Education, 38. doi:10.1123/jtpe.2018-0069

Keegan, R., Dudley, D., Bryant, A., Evans, J., Farrow, D., Lubans, D., . . . Barnett, L. (2019). Defining physical literacy: A modified Delphi method. Journal of Teaching in Physical Education, 38. doi:10.1123/ jtpe.2018-0264

Kriellaars, D., Cairney, J., Bortoleto, M., Kiez, T., Dudley, D., \& Aubertin, P. (2019). The impact of circus arts instruction in physical education on the physical literacy of children in grades 4 and 5. Journal of Teaching in Physical Education, 38. doi:10.1123/jtpe.2018-0269

Mandigo, J., Treadway, J., \& Lodewyk, K. (2019). Examining the impact of a Teaching Games for Understanding approach on the development of physical literacy using the Passport for Life assessment tool. Journal of Teaching in Physical Education, 38. doi:10.1123/jtpe.2018-0028

Shortt, C., Webster, C.A., Keegan, R., Egan, C.A., \& Brian, A. (2019). Operationally conceptualizing physical literacy: Results of a Delphi study. Journal of Teaching in Physical Education, 38. doi:10.1123/ jtpe.2018-0202 